\title{
JMASM39: Algorithm for Combining Robust and Bootstrap In Multiple Linear Model Regression (SAS)
}

Wan Muhamad Amir

University Science Malaysia, wmamir@usm.my

Mohamad Shafiq

University Science Malaysia, shafiqmat786@gmail.com

Hanafi A.Rahim

University Malaysia Terengganu, hanafi@umt.edu.my

Puspa Liza

University of Sultan Zainal Abidin, puspaliza@unisza.edu.my

Azlida Aleng

University Malaysia Terengganu, azlida_aleng@umt.edu.my

See next page for additional authors

Follow this and additional works at: http://digitalcommons.wayne.edu/jmasm

Part of the Applied Statistics Commons, Social and Behavioral Sciences Commons, and the Statistical Theory Commons

\section{Recommended Citation}

Amir, Wan Muhamad; Shafiq, Mohamad; A.Rahim, Hanafi; Liza, Puspa; Aleng, Azlida; and Abdullah, Zailani (2016) "JMASM39: Algorithm for Combining Robust and Bootstrap In Multiple Linear Model Regression (SAS)," Journal of Modern Applied Statistical Methods: Vol. 15 : Iss. 1 , Article 46.

DOI: $10.22237 /$ jmasm/1462077900

Available at: http://digitalcommons.wayne.edu/jmasm/vol15/iss1/46

This Algorithms and Code is brought to you for free and open access by the Open Access Journals at DigitalCommons@WayneState. It has been accepted for inclusion in Journal of Modern Applied Statistical Methods by an authorized editor of DigitalCommons@WayneState. 


\section{JMASM39: Algorithm for Combining Robust and Bootstrap In Multiple Linear Model Regression (SAS)}

Erratum

This paper was originally published in JMASM Algorithms \& Code without its enumeration, JMASM39.

Authors

Wan Muhamad Amir, Mohamad Shafiq, Hanafi A.Rahim, Puspa Liza, Azlida Aleng, and Zailani Abdullah 


\section{JMASM Algorithms and Code Algorithm for Combining Robust and Bootstrap In Multiple Linear Model Regression (SAS)}

\section{Wan Muhamad Amir \\ University of Science, Malaysia \\ Kelantan, Malaysia}

\section{Puspa Liza}

Universiti Sultan Zainal Abidin

Kuala Terengganu, Malaysia

\author{
Mohamad Shafiq \\ University of Science, Malaysia \\ Kelantan, Malaysia
}

Azlida Aleng

University Malaysia Terengganu

Kuala Terengganu, Malaysia

\section{Hanafi A. Rahim}

University Malaysia Terengganu

Kuala Terengganu, Malaysia

\section{Zailani Abdullah}

University Malaysia Kelantan

Kelantan, Malaysia

The aim of bootstrapping is to approximate the sampling distribution of some estimator. An algorithm for combining method is given in SAS, along with applications and visualizations.

Keywords: $\quad$ Multiple linear regression, robust regression and bootstrap method

\section{Introduction}

Multiple linear regression (MLR) is an extension of simple linear regression. Table 1 displays the data for multiple linear regression.

Table 1. Data template for multiple linear regression

\begin{tabular}{|c|c|c|c|c|c|c|}
\hline$i$ & $y_{i}$ & $x_{0}$ & $X_{\boldsymbol{i}}$ & $x_{12}$ & $\ldots$ & $x_{i p}$ \\
\hline 1 & $y_{1}$ & 1 & $X 11$ & $x 12$ & $\ldots$ & $x_{1 p}$ \\
\hline 2 & $y_{2}$ & 1 & $x_{21}$ & $x 22$ & $\ldots$ & $x_{2 p}$ \\
\hline$\vdots$ & $\vdots$ & $\vdots$ & $\vdots$ & $\vdots$ & & $\vdots$ \\
\hline$n$ & $y_{n}$ & 1 & $x_{n 1}$ & $x_{n 2}$ & $\ldots$ & $x_{n p}$ \\
\hline
\end{tabular}

Dr. Amir bin W Ahmad is an Associate Professor of Biostatistics. Email him at: wmamir@usm.my. Mohamad Shafiq Bin Mohd Ibrahim is a postgraduate student in the School of Dental Sciences. Email him at: shafiqmat786@gmail.com. 


\section{AMIR ET AL}

MLR is used when there are two or more independent variables where the model using population information is

$$
y_{i}=\beta_{0}+\beta_{1} x_{1 i}+\beta_{2} x_{2 i}+\beta_{3} x_{3 i}+\ldots+\beta_{k} x_{k i}+\varepsilon_{i}
$$

where $\beta_{0}$ is the intercept parameter and $\beta_{0}, \beta_{1}, \beta_{2}, \ldots, \beta_{k-1}$ are the parameters associated with $k-1$ predictor variables. The dependent variable $\mathbf{Y}$ is now written as a function of $k$ independent variables, $x_{1}, x_{2}, \ldots, x_{k}$.

The random error term is added to make the model probabilistic rather than deterministic. The value of the coefficient $\beta_{i}$ determines the contribution of the independent variable $x_{i}$, and $\beta_{0}$ is the $y$-intercept. (Ngo, 2012). The coefficients $\beta_{0}$, $\beta_{1}, \ldots, \beta_{k}$ are usually unknown because they represent population parameters. Below is the data presentation for multiple linear regression. General linear model in matrix form can be defined by the following vectors and matrices as below:

$$
\mathbf{Y}=\left[\begin{array}{c}
Y_{1} \\
Y_{2} \\
\vdots \\
Y_{n}
\end{array}\right], \mathbf{X}=\left[\begin{array}{ccccc}
1 & X_{11} & X_{12} & \cdots & X_{1, p-1} \\
1 & X_{21} & X_{22} & \cdots & X_{2, p-1} \\
\vdots & \vdots & \vdots & & \vdots \\
1 & X_{n 1} & X_{n 2} & \cdots & X_{n, p-1}
\end{array}\right], \boldsymbol{\beta}=\left[\begin{array}{c}
\beta_{0} \\
\beta_{1} \\
\vdots \\
\beta_{p-1}
\end{array}\right], \boldsymbol{\varepsilon}=\left[\begin{array}{c}
\varepsilon_{0} \\
\varepsilon_{1} \\
\vdots \\
\varepsilon_{p-1}
\end{array}\right]
$$

\section{Calculation for Linear Regression using SAS}

/* First we do simple Linear regression */ proc reg data = temp1;

model $\mathrm{y}=\mathrm{x}$;

run;

\section{Approach the MM-Estimation Procedure for Robust Regression}

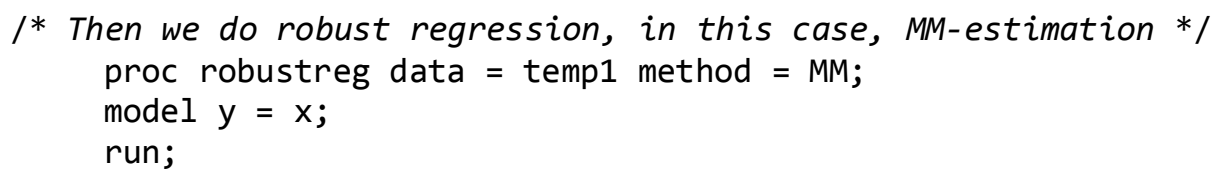

\section{Procedure for Bootstrap with Case Resampling $\boldsymbol{n}=\mathbf{1 0 0 0}$}

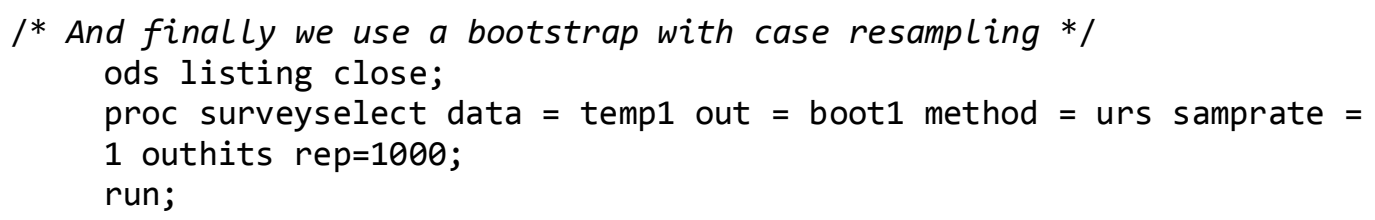




\section{ALGORITHM FOR COMBINING IN MULTIPLE LINEAR REGRESSION}

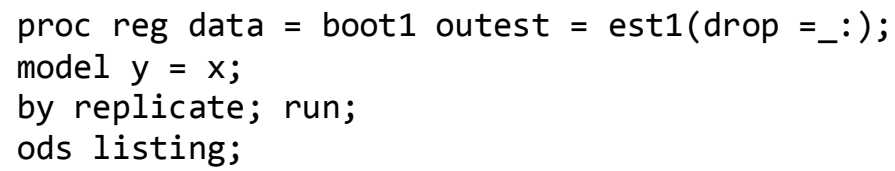

\section{An Illustration of a Medical Case}

\section{Case Study I: A Case Study of Triglycerides}

Table 2. Description of the variables

\begin{tabular}{rrr} 
Variables & Code & Description \\
\hline Triglycerides & $Y$ & Triglycerides level of patients $(\mathrm{mg} / \mathrm{dl})$ \\
Weight & $X 1$ & Weight $(\mathrm{kg})$ \\
Total Cholesterol & $X 2$ & Total cholesterol of patients $(\mathrm{mg} / \mathrm{dl})$ \\
Proconvertin & $X 3$ & Proconvertin $(\%)$ \\
Glucose & $X 4$ & Glucose level of patients $(\mathrm{mg} / \mathrm{dl})$ \\
HDL-Cholesterol & $X 5$ & High density lipoprotein cholesterol $(\mathrm{mg} / \mathrm{dl})$ \\
Hip & $X 6$ & Hip circumference $(\mathrm{cm})$ \\
Insulin & $X 7$ & Insulin level of patients $(\mathrm{IU} / \mathrm{ml})$ \\
Lipid & $X 8$ & Taking lipid lowering medication $(0=$ no, $1=$ yes $)$ \\
\hline
\end{tabular}

Sources: Ahmad and Ibrahim (2013), Ahmad, Ibrahim, Halim, and Aleng (2014)

\section{Algorithm for Combining Robust and Bootstrap in Multiple Linear Model Regression}

Title 'Alternative Modeling on Multiple linear regression';

Data Medical;

$\begin{array}{llllllllll}\text { Input } & Y & \text { X1 } & \text { X2 } & \text { X3 } & \text { X4 } & \text { X5 } & \text { X6 } & \text { X7 } & \text { X8; }\end{array}$

Datalines;

$\begin{array}{lllllllll}168 & 85.77 & 209 & 110 & 114 & 37 & 130.0 & 17 & 0 \\ 304 & 58.98 & 228 & 111 & 153 & 33 & 105.5 & 28 & 1 \\ 72 & 33.56 & 196 & 79 & 101 & 69 & 88.5 & 6 & 0 \\ 119 & 49.00 & 281 & 117 & 95 & 38 & 104.2 & 10 & 1 \\ 116 & 38.55 & 197 & 99 & 110 & 37 & 92.0 & 12 & 0 \\ 87 & 44.91 & 184 & 131 & 100 & 45 & 100.5 & 18 & 0 \\ 136 & 48.09 & 170 & 96 & 108 & 37 & 96.0 & 13 & 1 \\ 78 & 69.43 & 163 & 89 & 111 & 39 & 103.0 & 8 & 0 \\ 223 & 47.63 & 195 & 177 & 112 & 39 & 95.0 & 15 & 0 \\ 200 & 55.35 & 218 & 108 & 131 & 31 & 104.0 & 33 & 1 \\ 159 & 59.66 & 234 & 112 & 174 & 55 & 114.0 & 14 & 0 \\ 181 & 68.97 & 262 & 152 & 108 & 44 & 114.5 & 20 & 1 \\ 134 & 51.49 & 178 & 127 & 105 & 51 & 100.0 & 21 & 0 \\ 162 & 39.69 & 248 & 135 & 92 & 63 & 93.0 & 9 & 1\end{array}$




\section{AMIR ET AL}

$\begin{array}{lllllllll}96 & 56.58 & 210 & 122 & 105 & 56 & 103.4 & 6 & 0 \\ 117 & 63.48 & 252 & 125 & 99 & 70 & 104.2 & 10 & 0 \\ 106 & 66.70 & 191 & 103 & 101 & 32 & 103.3 & 16 & 0 \\ 120 & 74.19 & 238 & 135 & 142 & 50 & 113.5 & 14 & 1 \\ 119 & 60.12 & 169 & 98 & 103 & 33 & 114.0 & 13 & 0 \\ 116 & 36.60 & 221 & 113 & 88 & 60 & 94.3 & 11 & 1 \\ 109 & 56.40 & 216 & 128 & 90 & 49 & 107.1 & 13 & 0 \\ 105 & 35.15 & 157 & 114 & 88 & 35 & 95.0 & 12 & 0 \\ 88 & 50.13 & 192 & 120 & 100 & 54 & 100.0 & 11 & 0 \\ 241 & 56.49 & 206 & 137 & 148 & 79 & 113.0 & 14 & 1 \\ 175 & 57.39 & 164 & 108 & 104 & 42 & 103.0 & 15 & 0 \\ 146 & 43.00 & 209 & 116 & 93 & 64 & 97.0 & 13 & 0 \\ 199 & 48.04 & 219 & 104 & 158 & 44 & 97.0 & 11 & 0 \\ 85 & 41.28 & 171 & 92 & 86 & 64 & 95.4 & 5 & 0 \\ 90 & 65.79 & 156 & 80 & 98 & 54 & 98.5 & 11 & 1 \\ 87 & 56.90 & 247 & 128 & 95 & 57 & 106.3 & 9 & 0 \\ 103 & 35.15 & 257 & 121 & 111 & 69 & 89.5 & 13 & 0 \\ 121 & 55.12 & 138 & 108 & 104 & 36 & 109.0 & 13 & 0 \\ 223 & 57.17 & 176 & 112 & 121 & 38 & 114.0 & 32 & 0 \\ 76 & 49.45 & 174 & 121 & 89 & 47 & 101.0 & 8 & 0 \\ 151 & 44.46 & 213 & 93 & 116 & 45 & 99.0 & 10 & 1 \\ 145 & 56.94 & 228 & 112 & 99 & 44 & 109.0 & 11 & 0 \\ 196 & 44.00 & 193 & 107 & 95 & 31 & 96.5 & 12 & 0 \\ 113 & 53.54 & 210 & 125 & 111 & 45 & 105.5 & 19 & 0 \\ 113 & 35.83 & 157 & 100 & 92 & 55 & 95.0 & 13 & 0 \\ ; & & & & & & & & \\ \text { Run; } & & & & & & & & \\ & & & & & & & & 0\end{array}$

ods rtf file='results_ex1.rtf';

/* This first step is to make the selection of the data that have a significant impact with triglyceride levels. The next step is performing the procedure of modeling Linear regression model */

proc reg data= Medical;

model $\mathrm{Y}=\mathrm{X} 1 \quad \mathrm{X} 2 \mathrm{X} 3 \quad \mathrm{X} 4 \quad \mathrm{X} 5 \quad \mathrm{X} 6 \quad \mathrm{X} 7 \quad \mathrm{X} 8$;

run;

/* Then do robust regression, in this case MM-estimation */

proc robustreg data $=$ Medical method $=$ MM;

model $\mathrm{Y}=\mathrm{X} 1 \mathrm{X} 2 \mathrm{X} 3 \mathrm{X} 4 \mathrm{X} 5 \mathrm{X} 6 \mathrm{X7}$ X8/ diagnostics leverage; 


\section{ALGORITHM FOR COMBINING IN MULTIPLE LINEAR REGRESSION}

output out=robout $r=$ resid $s r=s t d r e s$;

run;

/* Use a bootstrap with case resampling */

ods listing close;

proc surveyselect data= Medical out=boot1 method=urs samprate=1 outhits rep = 50;

run;

/* And finally use a bootstrap with robust with case resampling */ proc robustreg data=boot1 method=MM plot=fitplot(nolimits) plots=all; model $\mathrm{Y}=\mathrm{X} 1 \quad \mathrm{X} 2 \times 3 \quad \mathrm{X} 4 \quad \mathrm{X} 5 \quad \mathrm{X} 6 \quad \mathrm{X} 7 \quad \mathrm{X} 8$;

run;

ods rtf close;

\section{Results from Original Data}

Below are the results from the analysis using the original data. The residual plots do not indicate any problem with the model. A normal distribution appears to fit our sample data fairly well. The plotted points form a reasonably straight line. In our case, the residual bounce randomly around the 0 line (residual vs. predicted value). This suggest that the assumption that the relationship is linear is reasonable. A higher R-squared value of 0.62 indicated how well the data fit the model and also indicates a better model.

Table 3. Parameter estimates for original data

Parameter Estimates

\begin{tabular}{rrrrrr}
\hline Variable & DF & Parameter Estimate & Standard Error & $\boldsymbol{t}$ Value & $\operatorname{Pr}>|\boldsymbol{t}|$ \\
\hline Intercept & 1 & -86.5654 & 102.93662 & -0.84 & 0.4070 \\
$x 1$ & 1 & -1.08598 & 0.95288 & -1.14 & 0.2634 \\
$x 2$ & 1 & -0.06448 & 0.21973 & -0.29 & 0.7712 \\
$x 3$ & 1 & 0.61857 & 0.36615 & 1.69 & 0.1015 \\
$x 4$ & 1 & 1.10882 & 0.33989 & 3.26 & 0.0028 \\
$x 5$ & 1 & -0.52289 & 0.57119 & -0.92 & 0.3673 \\
$x 6$ & 1 & 0.81327 & 1.38022 & 0.59 & 0.5601 \\
$x 7$ & 1 & 2.77339 & 1.25026 & 2.22 & 0.0343 \\
$x 8$ & 1 & 22.40585 & 14.51449 & 1.54 & 0.1331 \\
\hline
\end{tabular}


AMIR ET AL

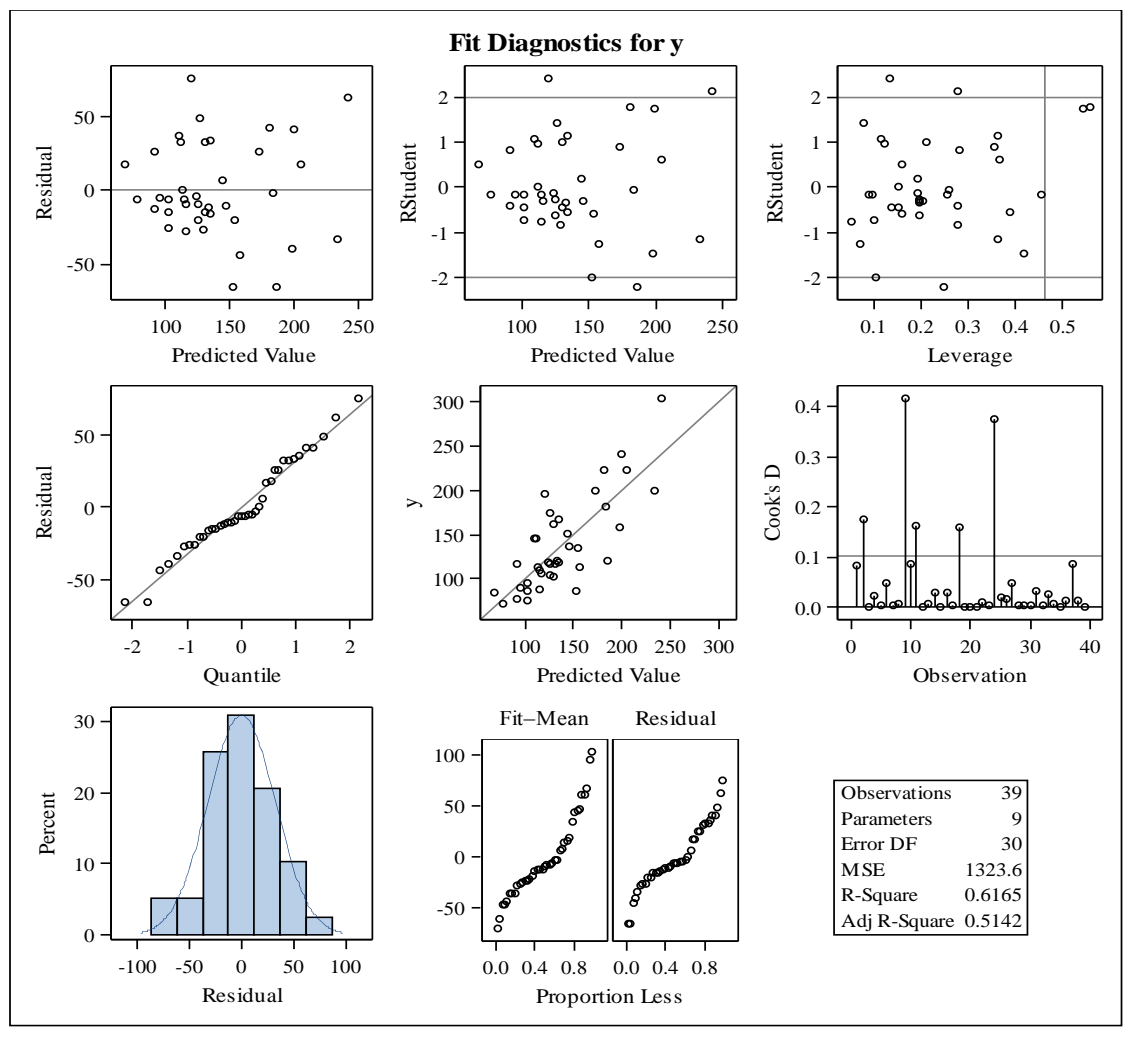

Figure 1. Fit diagnostic for $y$

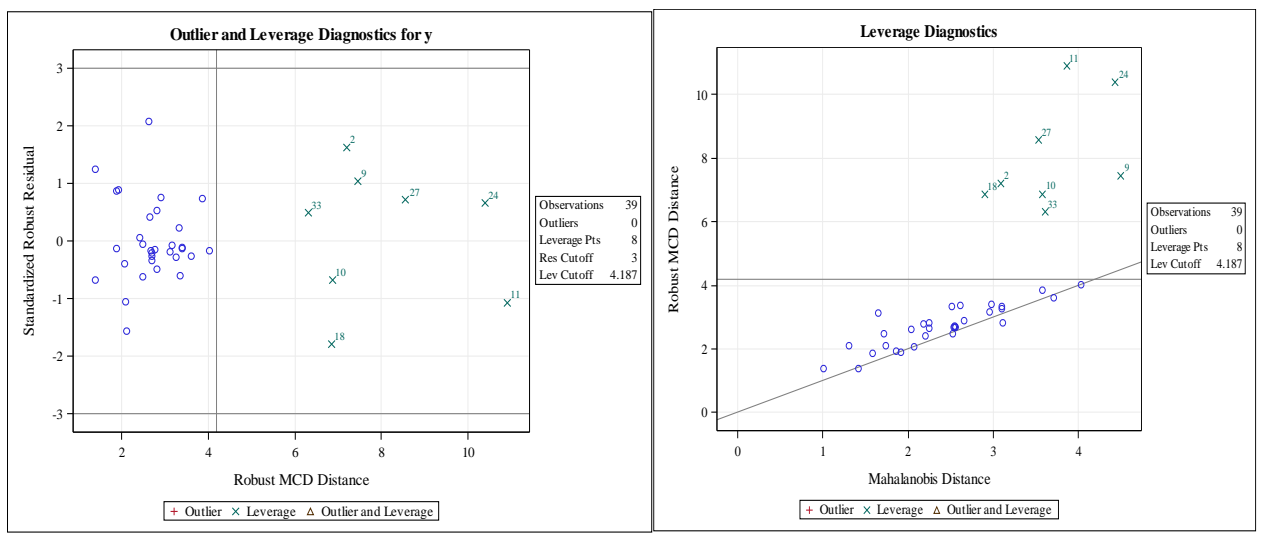

Figure 2. Outlier and Leverage Diagnostic for $y$ 


\section{ALGORITHM FOR COMBINING IN MULTIPLE LINEAR REGRESSION}

From Figure 2, we can see that there is no detection of outlier in observations. The leverage plots available in the SAS software are considered useful and effective in detecting multicollinearity, non-linearity, significance of the slope, and outliers (Lockwood \& Mackinnon, 1998). Both of figures above indicate that this sample have no peculiarity and a data entry have no error. Figure 2 presented a regression diagnostics plot (a plot of the standardized residuals of robust regression MM versus the robust distance). Observations 2, 9, 10, 11, 18, 24, 27 and 33 are identified as leverage points. Below is the results of bootstrapping with $n=50$ :

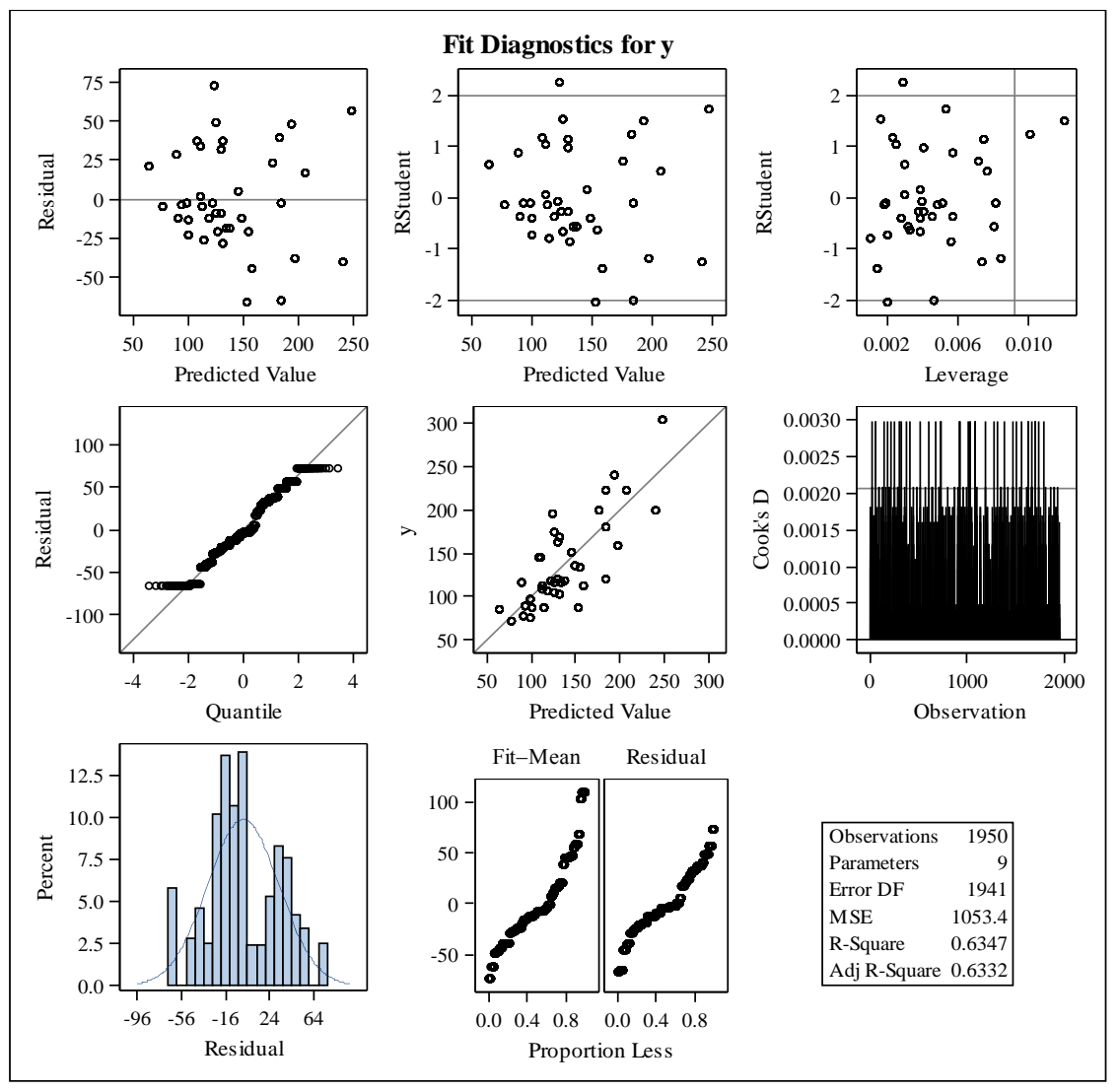

Figure 3. Fit diagnostic for $y$ after bootstrapping

Table 4 shows the results by using bootstrapping method. The aim of bootstrapping procedure is to approximate the entire sampling distribution of some estimator by resampling (simple random sampling with replacement) from the original data (Yaffee, 2002). The next step is to calculate the efficiency of the 


\section{AMIR ET AL}

bootstrap method with the original sample data. Table 5 summarize the findings of the calculated parameter.

Table 4. Parameter estimates using bootstrapping method

\begin{tabular}{|c|c|c|c|c|c|c|c|}
\hline \multirow{2}{*}{$\begin{array}{r}\text { Parameter } \\
\text { Intercept }\end{array}$} & \multirow{2}{*}{$\frac{\mathrm{DF}}{1}$} & \multirow{2}{*}{$\begin{array}{r}\text { Estimate } \\
-297.0810\end{array}$} & \multicolumn{3}{|c|}{$\begin{array}{l}\text { Parameter Estimates } \\
\text { Standard }\end{array}$} & Chi-Square & $\mathrm{Pr}>\mathrm{ChiSq}$ \\
\hline & & & 9.18120 & -315.0760 & -279.0860 & 1047.02 & $<0.0001$ \\
\hline$x 1$ & 1 & -1.3526 & 0.07910 & -1.5076 & -1.1977 & 292.69 & $<0.0001$ \\
\hline$x 2$ & 1 & 0.0286 & 0.01850 & -0.0077 & 0.0649 & 2.38 & 0.1227 \\
\hline$x 3$ & 1 & 0.0441 & 0.04360 & -0.0413 & 0.1295 & 1.03 & 0.3112 \\
\hline$x 4$ & 1 & 1.5405 & 0.03300 & 1.4759 & 1.6052 & 2182.31 & $<0.0001$ \\
\hline$x 5$ & 1 & 0.2976 & 0.04960 & 0.2004 & 0.3948 & 36.04 & $<0.0001$ \\
\hline$x 6$ & 1 & 2.6234 & 0.12240 & 2.3836 & 2.8632 & 459.66 & $<0.0001$ \\
\hline$x 7$ & 1 & 2.4174 & 0.10580 & 2.2100 & 2.6248 & 521.88 & $<0.0001$ \\
\hline$x 8$ & 1 & 24.6443 & 1.20480 & 22.2829 & 27.0057 & 418.39 & $<0.0001$ \\
\hline Scale & 0 & 27.6976 & & & & & \\
\hline
\end{tabular}

Table 5. Comparison of parameter estimates original sample and bootstrapping method

\begin{tabular}{rrrrrrrr} 
& \multicolumn{9}{c}{ Parameter Estimates } \\
Variables & \multicolumn{9}{c}{$\begin{array}{c}\text { Original sample } \\
\text { Parameter } \\
\text { Estimate }\end{array}$} & $\begin{array}{r}\text { Standard } \\
\text { Error }\end{array}$ & P value & \multicolumn{2}{c}{$\begin{array}{c}\text { Bootstrapping Method } \\
\text { Estimate }\end{array}$} & $\begin{array}{r}\text { Standard } \\
\text { Error }\end{array}$ & P value & $\begin{array}{r}\text { Efficiency } \\
\text { of } \\
\text { Parameter } \\
(\%)\end{array}$ \\
\hline Intercept & -86.56544 & 102.93662 & 0.4070 & -297.0810 & 9.1812 & $<0.0001$ & \\
$x 1$ & -1.08598 & 0.95288 & 0.2634 & -1.3526 & 0.0791 & $<0.0001$ & 24.55 \\
$x 2$ & -0.06448 & 0.21973 & 0.7712 & 0.0286 & 0.0185 & 0.1227 & 144.35 \\
$x 3$ & 0.61857 & 0.36615 & 0.1015 & 0.0441 & 0.0436 & 0.3112 & 92.87 \\
$x 4$ & 1.10882 & 0.33989 & 0.0028 & 1.5405 & 0.0330 & $<0.0001$ & 38.93 \\
$x 5$ & -0.52289 & 0.57119 & 0.3673 & 0.2976 & 0.0496 & $<0.0001$ & 156.91 \\
$x 6$ & 0.81327 & 1.38022 & 0.5601 & 2.6234 & 0.1224 & $<0.0001$ & 222.57 \\
$x 7$ & 2.77339 & 1.25026 & 0.0343 & 2.4174 & 0.1058 & $<0.0001$ & 12.83 \\
$x 8$ & 22.40585 & 14.51449 & 0.1331 & 24.6443 & 1.2048 & $<0.0001$ & 9.99 \\
\hline
\end{tabular}

\section{References}

Ahmad, W. M. A. W., \& Ibrahim, M. S. (2013). High density lipoprotein cholesterol predicts triglycerides level in three distinct phases of blood pressure. International Journal of Sciences: Basic and Applied Research, 10(1), 38-46. Retrieved from 


\section{ALGORITHM FOR COMBINING IN MULTIPLE LINEAR REGRESSION}

http://gssrr.org/index.php?journal=JournalOfBasicAndApplied\&page=article\&op =view\&path\%5B\%5D=1111\&path\%5B\%5D=1098

Ahmad, W. M. A. W., Ibrahim, M. S., Halim, N., \& Aleng, N. A. (2014). A study of triglycerides level in three distinct phases of human blood pressure: A case study from previous projects. Applied Mathematical Sciences, 8(46), 22892305. doi: 10.12988/ams.2014.42145

Lockwood, C. M., \& Mackinnon, D. P. (1998). Bootstrapping the standard error off the mediated effect. Proceedings of the $23 \mathrm{rd}$ Annual Meeting of SAS Users Group International. Cary, NC: SAS Institute, Inc.

Ngo, T. H. D. (2012). The steps to follow in a multiple regression analysis. Proceedings of the SAS Global Forum 2012 Conference (paper 333-2012). Cary, NC: SAS Institute Inc. Retrieved from http://support.sas.com/resources/papers/proceedings12/333-2012.pdf

Yaffee, R. A. (2002). Robust regression analysis: Some popular statistical package options. Statistics, Social Science, and Mapping Group, New York University, NY. Retrieved from http://web.ipac.caltech.edu/staff/fmasci/home/astro_refs/RobustRegAnalysis.pdf 